\title{
Numerical Modelling of Pump-Wavelength Dependence of High Harmonic Generation Efficiency
}

\author{
S. M. Senior, W. S. Brocklesby, and P. Horak \\ Optoelectronics Research Centre, University of Southampton, Southampton SO17 1BJ, UK
}

High harmonic generation (HHG) provides a table-top source of extreme ultraviolet and soft x-ray radiation. A range of pump laser systems are currently being considered by various research groups, and thus the pump-wavelength dependence of HHG is of significant practical interest. HHG efficiency is generally assumed to scale with pump-wavelength to the power of $\mathrm{P}$, where first experiments suggested $\mathrm{P}=-6.5$ [1] while theoretical models predict $\mathrm{P}=-4.7$ to -6.0 [2-5]. However, these investigations exploited simple single-atom models; insight into the efficiency of full experimental setups will therefore benefit future HHG laser designs.

We developed a numerical model that simulates in full the process of HHG in a gas-filled capillary. First, the nonlinear propagation of the pump through the capillary is calculated by solving a unidirectional pulse propagation equation. Next, using this simulated pump pulse, single-atom time-dependent Schrödinger equations are solved to calculate HHG throughout the capillary. Finally, the generated harmonics are propagated to the capillary output using the angular spectrum method. With this model we compare HHG of two geometries with typical Ti:sapphire pump laser parameters: a thin slice of argon, and an argon-filled capillary.

For the thin slice with pump wavelengths $820-1890 \mathrm{~nm}$ we found scaling with $\mathrm{P}=-4.4$ when the harmonic energies were integrated between 16 and $45 \mathrm{eV}$, with only small effect on the scaling when changing the upper limit. A more detailed analysis revealed a dependence of $\mathrm{P}=-6.2$ for longer pump wavelengths $(1500-1890 \mathrm{~nm})$, but $\mathrm{P}=-4.1$ for shorter wavelengths $(820-1500 \mathrm{~nm})$. By contrast, HHG in a 7-cm long capillary was found to scale with $\mathrm{P}=-3.4$ (800-1850nm), a much weaker wavelength dependence. We attribute this to nonlinear pump propagation distorting the pulse and to phase-matching effects over longer propagation distances.

Different scaling is again observed when the energy of a single harmonic is calculated instead of an integral over a broad harmonic spectrum. For example, considering the energy in the first harmonic above $20 \mathrm{eV}$, generation in the thin slice of gas yields $\mathrm{P}=-6(820-1890 \mathrm{~nm}), \mathrm{P}=-5.8(820-1500 \mathrm{~nm})$, and $\mathrm{P}=-7.1(1500-1890 \mathrm{~nm})$. For the simulations of the whole capillary the corresponding value is $\mathrm{P}=-4.1(800-1850 \mathrm{~nm})$.

Another effect that strongly influences HHG efficiency is the classical harmonic cutoff energy which scales as the square of the pump wavelength. Therefore, high-energy harmonics exhibit very different scaling with pump wavelength as they cross this classical threshold. In particular, for the first harmonic beyond $41 \mathrm{eV}$ no value of $\mathrm{P}$ provided a good fit to the simulated HHG efficiencies, neither for the thin slice nor the whole capillary.

In conclusion, our simulations highlight that the pump-wavelength dependence of HHG efficiency is a complex issue with many factors such as the exact experimental geometry, optical nonlinearity, phase matching, and classical cutoff playing an important role.

[1] A. D. Shiner et al. Phys. Rev. Lett. 103, 073902 (2009)

[2] J. Tate et al. Phys. Rev. Lett. 98, 013901 (2007)

[3] K. Schiessl et al. Phys. Rev. Lett. 99, 253903 (2007)

[4] M. V. Frolov et al. Phys. Rev. Lett. 100, 173001 (2008)

[5] E. L. Falcão-Filho et al. Opt. Express 17, 11217-11229 (2009) 